\title{
Nanoparticle targeting of Gram-positive and Gram-negative bacteria for magnetic-based separations of bacterial pathogens
}

\author{
Hoang D. Lu ${ }^{1} \cdot$ Shirley S. Yang ${ }^{1} \cdot$ Brian K. Wilson ${ }^{1} \cdot$ Simon A. McManus ${ }^{1} \cdot$ \\ Christopher V. H.-H. Chen ${ }^{1} \cdot$ Robert K. Prud'homme ${ }^{1}$
}

Received: 27 December 2016/Accepted: 30 January 2017/Published online: 24 February 2017

(c) The Author(s) 2017. This article is published with open access at Springerlink.com

\begin{abstract}
Antimicrobial resistance is a healthcare problem of increasing significance, and there is increasing interest in developing new tools to address bacterial infections. Bacteria-targeting nanoparticles hold promise to improve drug efficacy, compliance, and safety. In addition, nanoparticles can also be used for novel applications, such as bacterial imaging or bioseperations. We here present the use of a scalable block-copolymer-directed self-assembly process, Flash NanoPrecipitation, to form zinc(II)-bis(dipicolylamine) modified nanoparticles that bind to both Grampositive and Gram-negative bacteria with specificity. Particles have tunable surface ligand densities that change particle avidity and binding efficacy. A variety of materials can be encapsulated into the core of the particles, such as optical dyes or iron oxide colloids, to produce imageable and magnetically active bacterial targeting constructs. As a proof-of-concept, these particles are used to bind and separate bacteria from solution in a magnetic column. Magnetic manipulation and separation would translate to a platform for pathogen identification or removal. These magnetic and targeted nanoparticles enable new methods to address bacterial infections.
\end{abstract}

Keywords Nanoparticle $\cdot$ Targeting $\cdot$ Bacteria $\cdot$ Filtering · Antimicrobial resistance $\cdot$ Magnetic separations

Electronic supplementary material The online version of this article (doi:10.1007/s13204-017-0548-0) contains supplementary material, which is available to authorized users.

Robert K. Prud'homme

prudhomm@princeton.edu

1 Department of Chemical and Biological Engineering, Princeton University, Princeton, NJ 08540, USA

\section{Introduction}

Bacterial infections are major contributors to morbidity and have become increasingly difficult to manage due to the emergence of antimicrobial resistance. There are over 2 million cases of antibiotic resistant infections in the US each year, causing $\$ 20$ billion in excess healthcare costs, $\$ 35$ billion in societal costs, 8 million additional hospitalization days, and 23,000 deaths annually (Centers for Disease and Prevention 2013; World Health Organization 2015). While antimicrobial resistance rates have been steadily rising, the development of new drugs has been steadily decreasing. These trends provide the impetus to develop new and improved methods to treat bacterial infections (Holmes et al. 2016; Ventola 2015a, b; Viswanathan 2014; Wisplinghoff et al. 2004).

Nanoparticles (NPs) are emerging as promising tools to help mitigate the rise of antimicrobial resistance (Zhang et al. 2010). NPs can encapsulate and deliver antimicrobials to improve drug pharmacokinetic and pharmacodynamics profiles, ultimately resulting in improved treatment outcomes (Peer et al. 2007; Zhang et al. 2008). Sustained drug release formulations decrease dosing frequencies and simplify dosing regimens, which can improve patient compliance and subsequently reduce resistance development rates from mismanaged drug use (Osterberg and Blaschke 2005; Wang et al. 2011). Targeted NPs can be especially effective at improving therapies by binding to and accumulating at sites of infections (Singh and Lillard 2009). Targeted NP delivery elevates the local concentrations of antibiotics in close proximity to bacteria, which increases drug bacteriostatic and bactericidal activities. Targeted delivery can also reduce off-target side-effects, and enable or revive the use of older-generation antibiotics that have been previously disregarded due to toxicity 
concerns (Falagas et al. 2008). In addition, targeted NPs can be used to image or phenotype infections by delivering imaging contrast agents (van Oosten et al. 2015).

NP targeting can be performed by modifying NP surfaces with functional groups that bind specifically to bacterial cell wall components. Targeting ligands include vancomycin which targets peptidoglycans found on Grampositive bacteria, polymyxin which targets lipopolysaccharides found on Gram-negative bacteria, or zinc(II)bis(dipicolylamine) (ZnDPA) which targets phosphatidylserine found on the surface of both Gram-positive and Gram-negative bacteria (Choi et al. 2013; Hanshaw and Smith 2005; Kell et al. 2008; Wong et al. 2015). Importantly, these ligand targets are not found on the surface of healthy mammalian cells, which allows for specific binding and imaging of bacteria with minimal background signals when using these targeting ligands in vivo (Rice et al. 2015; van Oosten et al. 2013). Recent novel uses of targeted NPs include the binding and magnetic separation of bacteria from solution to treat bacterial sepsis, by passing NPs through custom-made microfluidic devices (Lee et al. 2014). However, the use of microfuidics for the filtering of blood may be infeasible due to he large blood volumes and flow rates required for processing, relative to the volumes and flow rates ammenable to microfluidic processing. While NP targeting has been long appreciated, and has been employed in high-priced cancer treatments, the translation of NP-antibiotic constructs has been hindered due to the high cost of most NP formation processes relative to the low price of antibiotic therapies (Bartlett et al. 2013; Piddock 2012). In addition, the use of microfluidics for the filtering of blood may be unfeasible due to the large blood volumes and flow rates required for processing, relative to the volumes and flow rates permitted in microfluidic devices. The development of new bacteriatargeting NPs, formed by scalable NP production processes and that can be applied in scalable methods, can help translate new ways of combatting antibiotic resistant infections.

Flash NanoPrecipitation (FNP) is a continuous and scalable process for forming NPs that is ideally suited for industrial-scale and low-cost production of targeted encapsulated therapeutics (Johnson and Prud'homme 2003a, b; Pustulka et al. 2013; Shen et al. 2011; Weissmueller et al. 2016; Zhu 2014). In this technique, therapeutic actives are precipitated by rapid micromixing with an antisolvent in the presence of amphiphilic block copolymers. Block copolymers absorb onto precipitating actives to form sterically stabilized NPs with high drug loads up to $>75 \%$ weight. Polyethylene glycol (PEG) is typically used as the hydrophilic section of the blockcopolymer, to form a dense brush layer on the NP surface to confer water dispersability, resistance to opsonization, and long circulation times in vivo. The use of PEG endgroups modified with cell binding ligands results in NPs decorated with targeting groups on the NP surface (D'Addio et al. 2012, 2013). Importantly, this method allows particles to be formed with precise fractions of NP ligand PEG chain densities, using known blends of targeted and non-modified block copolymers during particle formation. This contrasts to the previous work that utilizes the conventional methods of reacting targeting ligands to functional groups on premade particles. Functionalization of pre-made NPs presents difficulties with validation of uniform ligand density on nanoparticle surfaces and difficulties in scaleup of the conjugation reactions to commercial scale. This is especially important for optimizing cell targeting, as increasing ligand densities can improve binding in some instances, but excessively high-ligand densities can also result in decreased cell binding (Allen 2002; Cabral et al. 2011; Cheng et al. 2012; D'Addio et al. 2013; Elias et al. 2013; Kathleen et al. 2007; Lee et al. 2010). The FNP process enables the simultaneous encapsulation of several hydrophobic actives, including imaging modalities and hydrophobic drugs, to create theranostic NPs for novel antimicrobial treatments ( $\mathrm{Lu}$ et al. 2015, 2016, 2017; Pansare et al. 2012, 2014). We here present the development and characterization of FNPbased NPs that target Gram-negative and Gram-positive bacteria, and demonstrate proof-of-concept applications where bacteria are separated from media using commercially available and easily scalable magnetic separation setups. The development of these FNP particles adds new tools for managing antibiotic resistant infections.

\section{Experimental}

\section{Materials}

$1.6 \mathrm{kDa}$ polystyrene-block-5 kDa polyethylene glycol (PS$b$-PEG, Polymer Source Inc.), $1.8 \mathrm{kDa}$ polystyrene homopolymer (PS, Polymer Source Inc), alpha tocopherol (VitE, Sigma-Aldrich), 6-8 kDa MWCO regenerated cellulose membrane (Specra/Por, Spectrum Labs), Lennox LB Broth (Fisher BioReagents), and magnetic MidiMACS column and separators (Miltenyi Biotec) were used as supplied. Ettp-5 fluorophore and ZnDPA with a carboxylic acid handle were synthesized as previously described (Lee et al. 2014; Pansare et al. 2014). PS- $b$-PEG was converted into PS- $b$ PEG modified with an amine (PS- $b$-PEG- $\mathrm{NH}_{2}$ ) functional group, and conjugated with ZnDPA via carbodiimide coupling to form PS- $b$-PEG-ZnDPA (Supplementary methods). Hydrophobic iron oxide (FeOA) was formed through the oxidation of iron salts and the addition of an oleic acid coat on iron oxide colloids (Supplementary methods). 


\section{Nanoparticle (NP) formation and characterization}

NPs with defined levels of surface ZnDPA or amine functionalities were formed through Flash NanoPrecipitation (FNP) processing as previously described (Johnson and Prud'homme 2003a; D'Addio et al. 2013). In brief, PS, Ettp5 dye, VitE, FeOA, PS- $b$-PEG, PS- $b$-PEG-ZnDPA, and PS- $b$ PEG-NH $\mathrm{N}_{2}$ were dissolved at defined compositions in THF, and rapidly micromixed with an equivalent volume of $100 \mathrm{mM}$ zinc nitrate within a confined impingement jet mixer, and diluted tenfold in a water collection bath. Particles with $0-100 \%$ ZnDPA surface functionalities were created using defined blends of PS- $b$-PEG/PS- $b$-PEG-ZnDPA in feed streams while keeping core-components constant. PS was included as a nanoparticle core bulking material, and Ettp5 was included as a fluorescent tracer and as an example active pharmaceutical ingredient. VitE was used as a core bulking material in particles loaded with magnetically active FeOA due to improve miscibility between oleic acid coated FeOA and VitE. The compositions of the particles formed are summarized in Table S1-S3.

Particle sizes and surface properties were assessed with dynamic light scattering analysis (Malvern Zetasizer Nano, Malvern Instruments). Size distributions were determined with backscattering measurements using $632 \mathrm{~nm}$ illumination, and displayed as intensity-weighted distributions. Zeta distributions were measured and analyzed with the Smoluchowski method after tenfold dilution into deionized water.

\section{Nanoparticle cell binding and quantification}

Overnight stationary cultures of Escherichia coli (K-12 MG1655), Staphylococcus aureus (Newman), and Pseudomonas aeruginosa (PA14) grown in LB were diluted 100 -fold in the same media, and grown to late exponential $\mathrm{OD}_{600}=1-1.2$ density cultures. Late exponential growth stage cells were inoculated with NPs by diluting nanoparticles tenfold into cell cultures $\left(\sim 100 \mu \mathrm{g} \mathrm{mL}^{-1} \mathrm{NP}\right.$ concentration) and incubation for $30 \mathrm{~min}$ at room temperature. Cells and bound NPs were harvested and separated from unbound NPs by centrifugation at $10,000 \mathrm{~g}$ for $1 \mathrm{~min}$. Unbound particles in media supernatant were aspirated, and cell pellets were resuspended in fresh LB media. Cells were washed twice, and collected for bound dye quantification via fluorescence microscopy. To quantify the extent of NP binding to cells, cell fluorescence was determined by reading the EttP5 fluorescence via flow cytometry (BD LSR-II Flow Cytometer, Becton-Dickinson) through excitation/emission in the Alexa-700 channel. For visual assessment of cell binding, washed cells with bound NPs were placed on glass slides and imaged with brightfield and fluorescence microscopy (Supplementary methods).

\section{Magnetic separation of bacteria}

Magnetic columns were decorated with ZnDPA surfacemodified FeOA NPs and utilized to separate bound cells using a modified version of the manufacturers Miltenyi Biotec MACS LD Column cell separation protocol. Cell separation columns were magnetized through placement on a MidiMACS Separator magnet and immobilized on a MACS MultiStand. Columns were primed with ten column volumes (CV) of LB, and loaded with $1.5 \mathrm{mg}$ of NPs. Unbound particles were discarded and $1 \mathrm{~mL}$ of exponential phase $E$. coli diluted to $\mathrm{OD}_{600}=0.02\left(\sim 2 \times 10^{7}\right.$ cells $)$ was added to the column, and unbound cells were collected. Columns were washed with $40 \mathrm{CV}$ of $\mathrm{LB}$ and effluent washed cells were collected and pooled with unbound cells. Cells were serially diluted and plated on LB-agar, whereupon colony forming unit titers were determined after plating onto LB plates and overnight growth. Cells were plated at the same time at the end of each series of experiments. For serial filtration experiments, three primed and NP loaded columns were prepared as described above. Pooled unbound and effluent washed cells from a single column were used to load the subsequent columns.

\section{Results and discussion}

\section{Nanoparticle formation}

NPs loaded with Ettp5 fluorophore were formed with ZnDPA surface functionalization at $0,6.3,13,25,50,75$, and $100 \%$ (i.e., percent of the PS-PEG stabilizing block copolymer with terminal ZnDPA ligands) using FNP. These NPs were formed by simply changing the blends of PS- $b$-PEG/PS- $b$-PEG-ZnDPA used during NP assembly (Fig. 1, Table S1). Particles at all surface functionalizes exhibited similar and narrow size distributions, and all particles had mean diameters near $\sim 120 \mathrm{~nm}$ (Fig. 2a, b). Dynamic light scattering results between all formulations attest to how the addition of ZnDPA had minimal effect on particle size, even at maximum surface functionalization. For example, $100 \%$ ZnDPA surface-modified NPs are $118 \pm 4 \mathrm{~nm}$ in diameter, while unmodified NPs are $108 \pm 5 \mathrm{~nm}$ in diameter. In addition, a linear regression displays no observed relationship between NP size and surface modification levels $\left(R^{2}<0.002\right)$. Thus, cell attachment can be attributed to ligand conjugation, rather than changes in nanoparticle size with changing ligand modification. The small size of ZnDPA allows for the formation of NPs with minimal impact on NP size, and is advantageous in contrast to large targeting moieties, such as antibodies, that cannot be grafted at the same PEG chain modification density. 


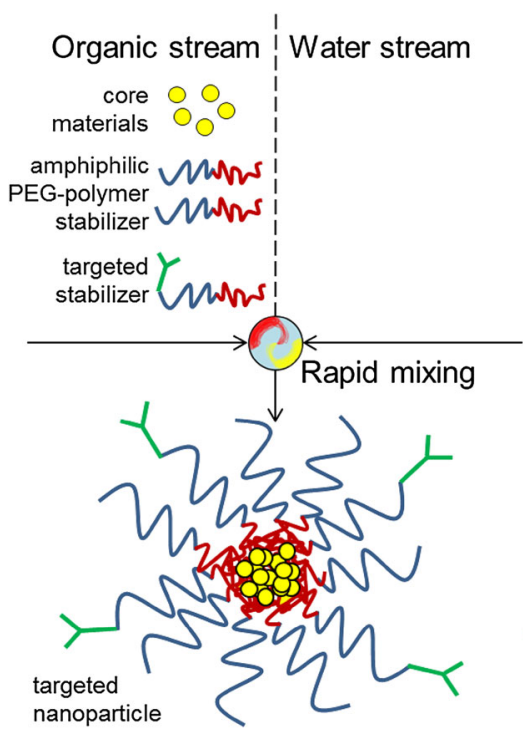

Fig. 1 Formation of bacterial targeting nanoparticles through Flash NanoPrecipitation. Core materials, amphiphilic block copolymers, and ligand conjugated block copolymers are dissolved in an organic solvent and rapidly impinged with water to initiate the precipitation of hydrophobic core materials. Block copolymers arrest the growth of

While NP size remained similar between all modification levels, the zeta potentials of NPs varied with modification extent (Fig. 2c, d). Unmodified NPs have a small zeta potential of $-1.1 \pm 4.3 \mathrm{mV}$, while $100 \%$ modified NPs have a zeta potential of $16.9 \pm 3.8 \mathrm{mV}$. The increase of zeta potential with higher modification is consistent with increased levels of ZnDPA presentation at the NP surface, as ZnDPA would exhibit a positive charge. Notably, the zeta potential and surface modifications are directly and linearly correlated $\left(R^{2}>0.99\right)$. These results highlight how particles with clearly defined and quantitative surface properties can be formed by simply changing block copolymer compositions during FNP. This is advantageous in how ligand conjugation can be performed and validated prior to particle formation, in contrast to grafting-on approaches where targeting moieties are conjugated onto premade NPs, and where there may be relatively poor batch to batch uniformity, especially for syntheses at larger scale. Altogether, NP size and zeta properties demonstrate that FNP can be used to form NPs of comparable sizes with tunable surface chemistry and targeting properties.

\section{Cell binding}

To determine if ZnDPA-decorated FNP particles would bind to bacteria, bacterial cells were incubated with unmodified and with 50\% ZnDPA-modified NPs. E. coli and $P$. aeruginosa was used to model binding to Gramnegative bacteria, and $S$. aureus was used to model

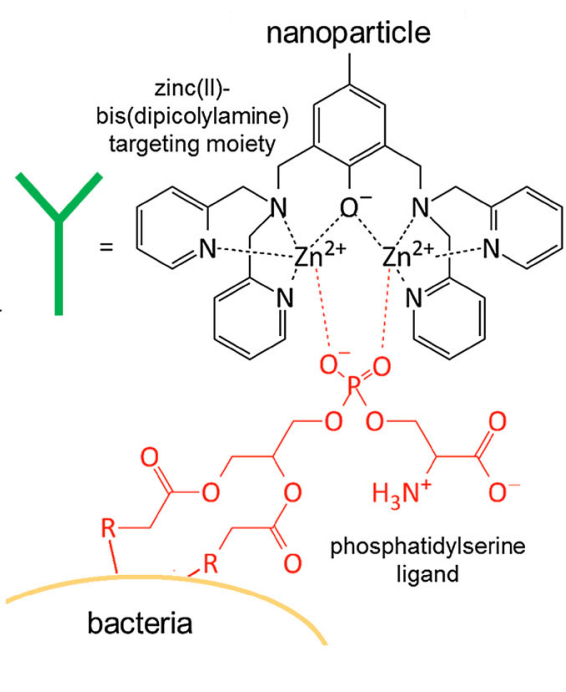

the hydrophobic materials and form sterically stabilized nanoparticles. The use of block copolymers modified with zinc(II)-bis(dipicolylamine) results in the formation of ligand surface functionalized nanoparticles that bind to phosphatidylserine targets, which are found on the surface of Gram-positive and Gram-negative bacteria

binding to Gram-positive bacteria. While all three cell types appeared as off-yellow pellets when harvested after inoculation with unmodified nanoparticles that encapsulate green Ettp5 dye, all three cell types appeared green after treatment with 50\% ZnDPA NPs that encapsulate Ettp5 (Fig. 3a). The E. coli and $S$. aureus cell pellets were dark green colored, while that of $P$. aeruginosa was lightly green colored, which suggests differences in binding levels among the bacterial species. To validate that particles were binding to bacteria, washed cells were additionally flame-fixed and imaged on glass slides (Fig. 4, Figure S1). Bright field images of fixed $E$. coli and $S$. aureus incubated with either unmodified or modified bacteria show characteristic rod or spherical shapes, respectively. Fluorescent imaging of the same samples shows strong fluorescence signals in samples incubated with 50\% ZnDPA NPs, while samples incubated with unmodified NPs exhibited background levels of fluorescence. Importantly, fluorescence signals are spatially co-registered with the locations of the bacteria as determined with bright field images, for both Gram-positive E. coli and Gram-negative $S$. aureus. These results attest that $\mathrm{ZnDPA}$ functionalized particles formed with the scalable and rapid FNP process can be utilized to bind both Gram-positive and Gram-negative bacteria.

To study how varying NP ZnDPA surface density affects binding, cells were incubated with NPs exhibiting $0,6.3,13,25,50$, and 100\% ZnDPA-modified NPs and characterized via flow cytometry (Fig. 5). Particles below 

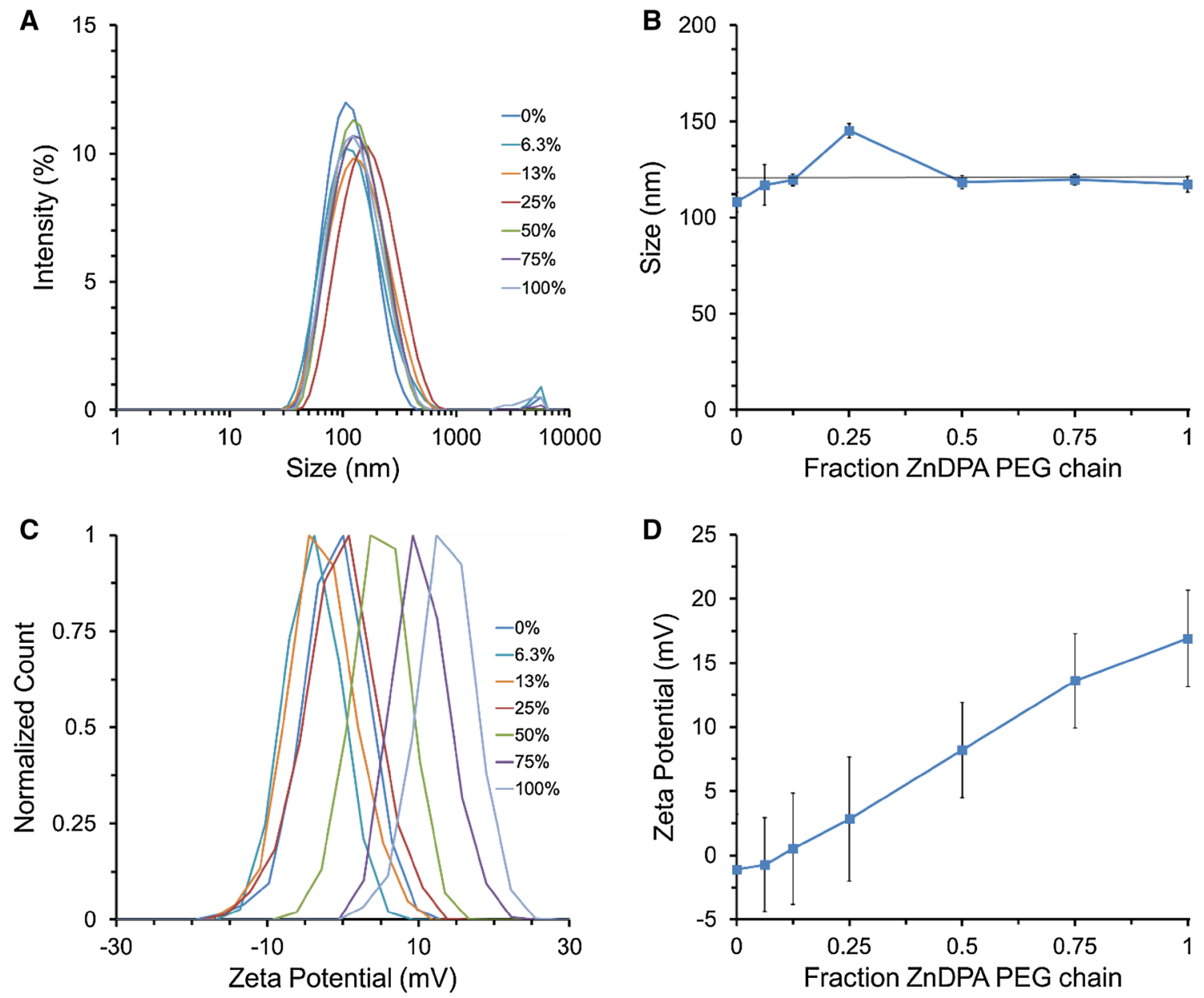

Fig. 2 Formation of ZnDPA-modified nanoparticles. a Intensityweighted dynamic light scattering size distributions and $\mathbf{b}$ diameter averages of particles made with various $\mathrm{ZnDPA}$ modifications.

c Normalized zeta potential distributions and $\mathbf{d}$ averages of particles made with various $\mathrm{ZnDPA}$ modifications. Compositions of particles are detailed in Table $\mathrm{S} 1$
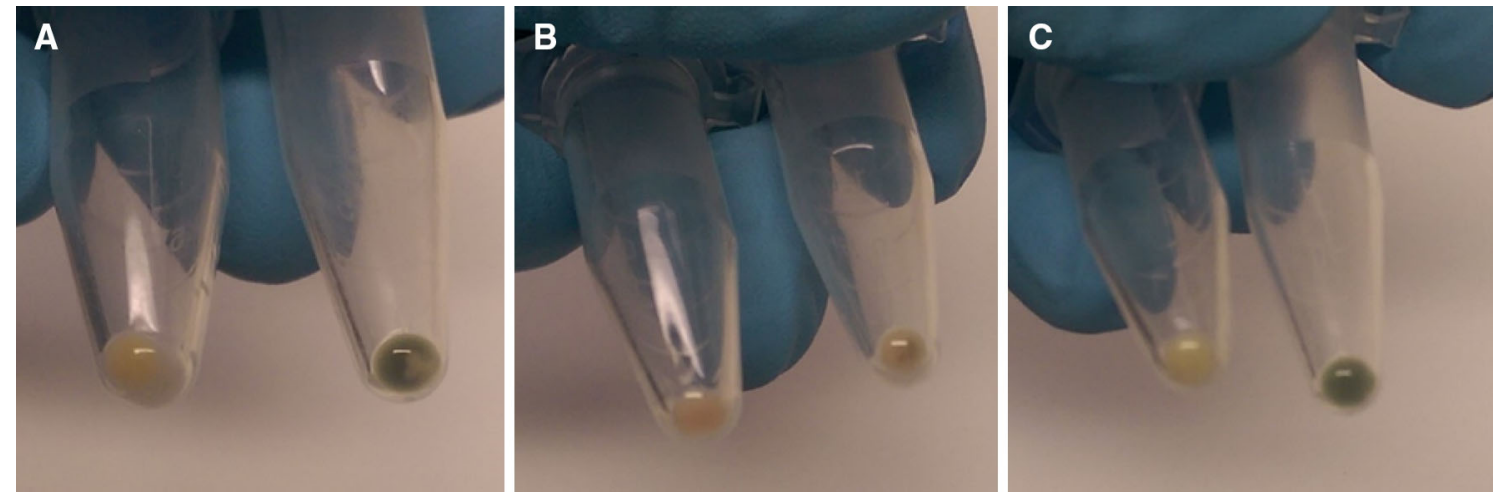

Fig. 3 Image of bacteria incubated with Flash NanoPrecpitationbased nanoparticles. Pellets of a Escherichia coli, b Pseudomonas aeruginosa, and c Staphylococcus aureus after grown to exponential

25\% ZnDPA modification exhibited minimal cell binding, while particles above $25 \%$ exhibited noticeable binding. For example, S. aureus incubated with 50\% ZnDPA NPs displayed a 33.5-fold higher mean fluorescence than those phase and inoculation with unmodified nanoparticles encapsulating a green Ettp5 dye (left), and inoculation with 50\% ZnDPA-modified nanoparticles encapsulating a green Ettp5 dye (right)

incubated with unmodified NPs, while that of E. coli was 16.7-fold higher, and that of $P$. aeruginosa was 6.2 -fold higher. These results are consistent with qualitative observations that $P$. aeruginosa cell pellets were less 

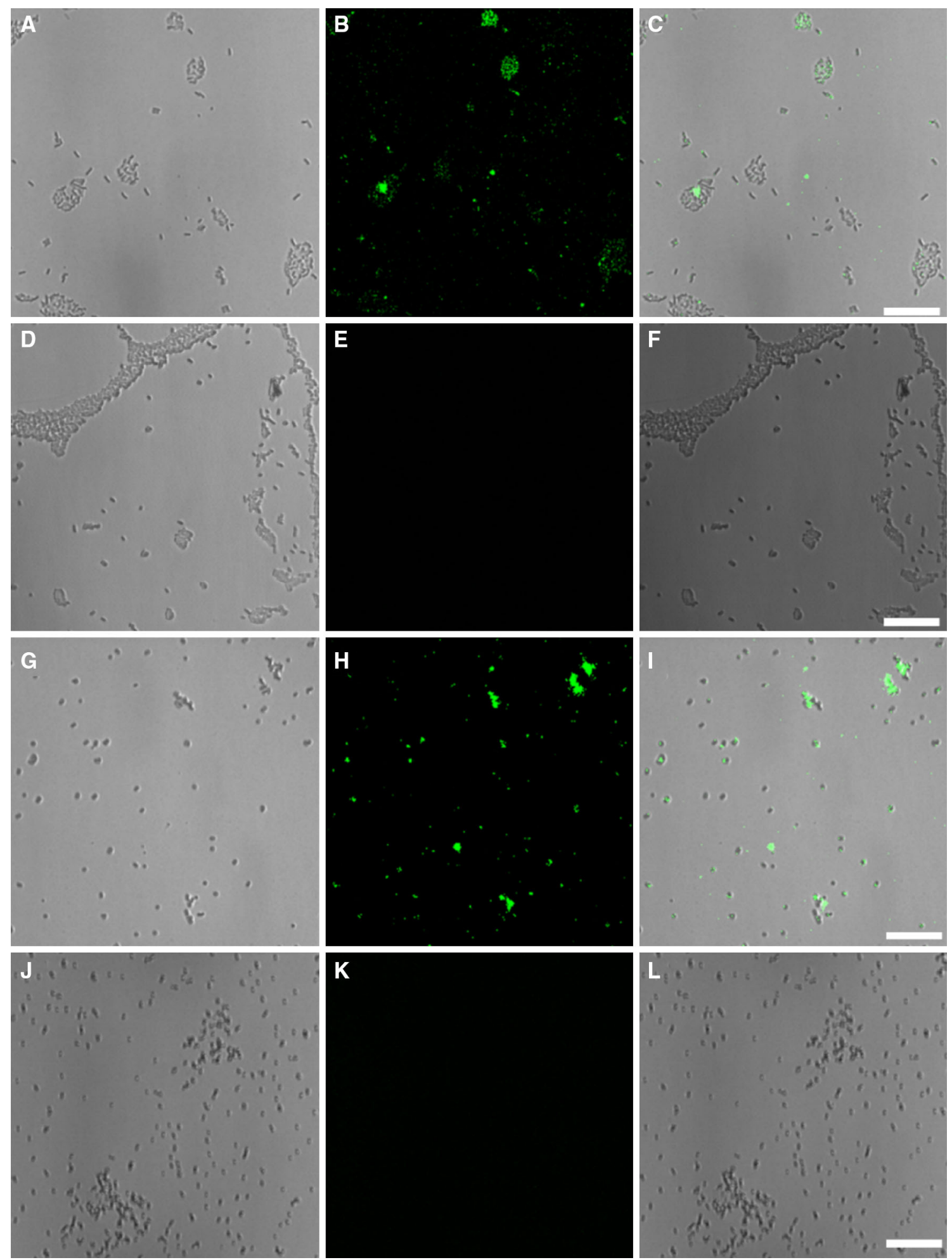

Fig. 4 Microscopy images of nanoparticle-binding to bacteria. Brightfield, fluorescence, and overlay images, respectively, of Gram-negative Escherichia coli incubated with a-c 50\% ZnDPAmodified NPs and $\mathbf{d}-\mathbf{f}$ unmodified ZnDPA NPs. Brightfield, fluorescence, and overlay images, respectively, of Gram-positive Staphylococcus aureus incubated with $\mathbf{g}-\mathbf{i}$ 50\% ZnDPA-modified NPs and $\mathbf{j}-\mathbf{l}$ unmodified ZnDPA NPs. Scale bar is $50 \mu \mathrm{m}$ 

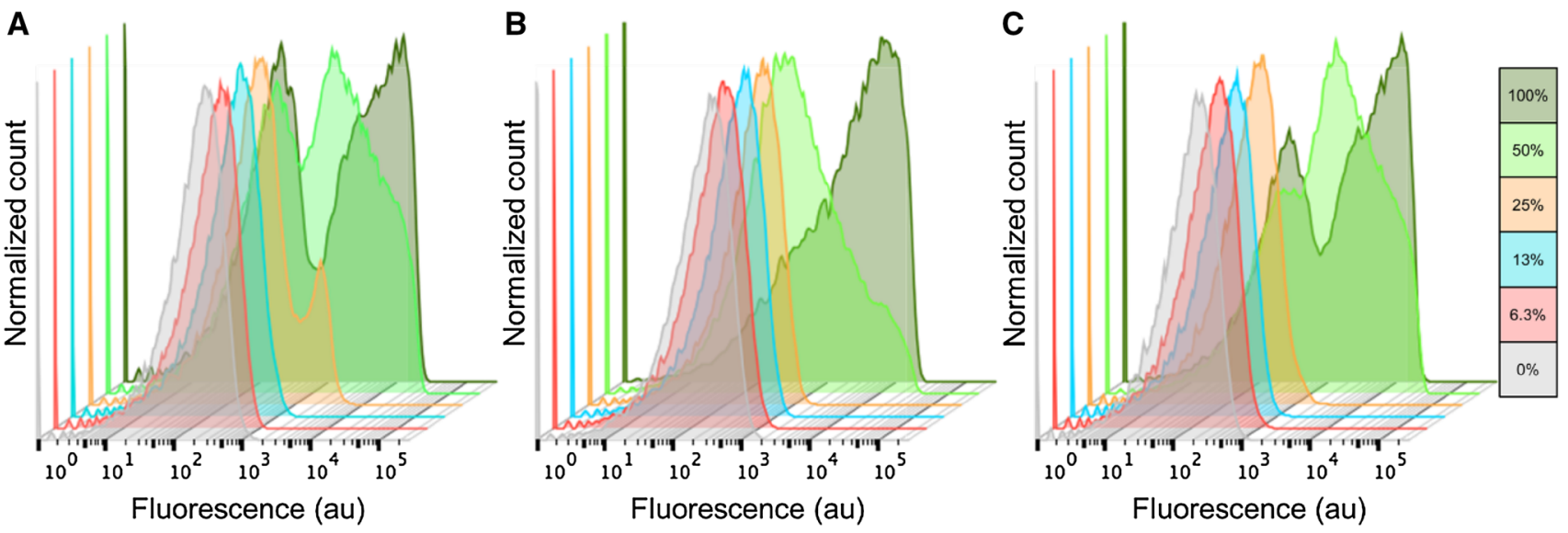

Fig. 5 Fluorescence distribution of bacteria incubated with ZnDPA nanoparticles. Normalized count distribution of fluorescent a $E$. coli $\mathbf{b} P$. aeruginosa and $\mathbf{c} S$. aureus after binding with 100, 50, 25, 13, 6.3, and $0 \%$ modified ZnDPA nanoparticles determined by flow cytometry

green than that of $E$. coli and $S$. aureus after incubation with ZnDPA-modified NPs. The exact mechanism for binding differences is interesting and should be investigated further, but may rooted in differences between cell membrane structure, cell morphology, or surface ligand densities among the different cell lines tested (Brown et al. 2015; Silhavy et al. 2010). These results also show that sufficient ZnDPA modification is required for NP binding to cells. Increased ZnDPA modification results in elevated local concentrations of ligands on NP surfaces, which can lead to increased NP avidity to cells.

To demonstrate that the ZnDPA targeting was not solely due to its cationic charge, analogous Ettp5 encapsulated NPs with various levels of amine functionality were made (Figure S1). These particles also exhibit a zeta potential that is linearly correlated with the amine modification extent, and are positive at high modifications due to the amine group (Figure S2). However, when 6.3, 13, 25 and $100 \%$ amine-modified NPs were incubated with E. coli, the binding to cells was insignificant. This demonstrates the specificity of the ZnDPA targeting moiety over simply positively charged nanoparticles in LB media. These results are consistent with the previous reports that $\mathrm{ZnDPA}$ displays a strong nanomolar range affinity to phosphatidylserine ligands even in high salinity buffers, whereas simple charge interactions can be screened due to ionic strength (Plaunt et al. 2014).

\section{Magnetically active NPs}

One major advantage of the FNP process is the ability to concurrently encapsulate actives in the core of NPs. Affinity and magnetic-based separation of bacteria have recently been identified as a potentially viable method to treat sepsis without the use of antimicrobials (Lee et al.
2014). To assess if ZnDPA-modified NPs can be used for analogous applications, hydrophobic $\mathrm{Fe}_{3} \mathrm{O}_{4}$ iron oxide colloids were synthesized and modified with a hydrophobic oleic acid coat such that iron oxide may be encapsulated into the core of the particles (Supplementary methods). When added to FNP formulation streams, stable particles with narrow size distributions approximately $\sim 150 \mathrm{~nm}$ were formed (Fig. 6a, Supplementary Table 3). The increased size of iron oxide loaded particles over nonloaded particles, that are $\sim 120 \mathrm{~nm}$, reflects the inclusion and sequestration of iron oxide in the particle core. Particle ZnDPA or amine surface modification, at either 50 and $100 \%$, did not affect particle size. Transmission electron microscopy images of 50 and $100 \%$ modified ZnDPA NPs with iron oxide cores reveal that the materials formed are symmetric and spherical particles with iron colloids homogenously dispersed in the particle core (Fig. 6b, c). TEM images of nanoparticles without an iron oxide core could not be imaged, due to the low electron density of polymeric materials in comparison to iron oxide. These results demonstrate that iron oxide colloids can be encapsulated into surface functionalized particles with the FNP method.

\section{Batch bacterial magnetic filtering}

While custom-made microfluidic devices have previously been used to magnetically filter bacteria from liquid samples, the application of customized microfluidic devices to filter large volumes of blood in clinical settings may be difficult to translate. To explore if FNP NPs can be used to filter bacteria in a scalable manner, iron oxide (FeOA) loaded NPs were incubated with $E$. coli as noted previously above and passed through a commercially available MidiMACS magnetic column. Cells that passed through the

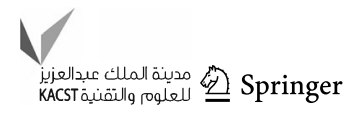



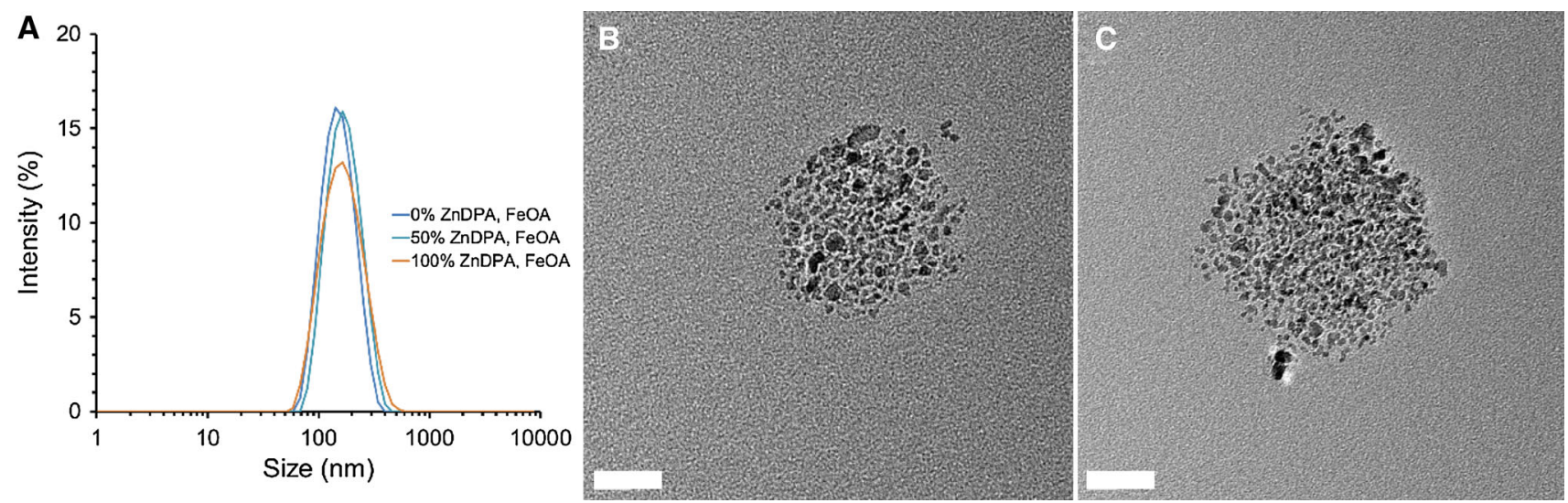

Fig. 6 Formation of iron oxide loaded NPs. a Intensity-weighted dynamic light scattering size distributions and transmission electron microscopy images of b $50 \%$ modified ZnDPA iron oxide NPs and c 100\% modified ZnDPA iron oxide NPs. Scale bar $40 \mathrm{~nm}$

column were collected and pooled with cells that were washed with excess media. After $\sim 20$ million cells were incubated with $50 \%$ ZnDPA-modified FeOA NPs and passed through the magnetic column, a total of $2.8 \pm 0.1$ million colony forming units were collected for a filtering efficiency of $86 \%$. In contrast, when cells were incubated with unmodified $\mathrm{FeOA}$ NPs, $17.0 \pm 4.2$ million colony forming units were formed, which is consistent with little no bacterial filtering when unmodified particles are used. These results demonstrate the ability to magnetically separate bacteria using ZnDPA NPs with a commercial magnetic separation process in a batch incubation process.

\section{Continuous bacterial magnetic filtering}

The development of a continuous and scalable method of magnetically isolating bacteria from solution would make bacterial separation processes easier to translate to the clinic. This would enable a fixed-bed filtration process where the column could be regnerated by loading and releasing NPs from the bed. Towards this end, we investigated to what extent magnetic columns pre-loaded with ZnDPA-FeOA NPs can be utilized for bacterial filtering applications. In this method, 1 $\mathrm{mL}$ of formed FeOA NPs was first passed through magnetic columns and washed, whereupon $\sim 20$ million E. coli cells were subsequently filtered through the column. When unmodified FeOA NPs were used, $25.1 \pm 3.8$ million CFU were collected in the flowthrough and wash cell streams, while $17.3 \pm 1.9$ million $\mathrm{CFU}$ and $3.84 \pm 1.3$ million $\mathrm{CFU}$ were collected with $50 \%$ ZnDPA and $100 \%$ ZnDPA-modified NPs, respectively (Fig. 7). Although $\sim 20$ million cells were included in the column based on optical density, CFU slightly greater than 20 million was collected, since bacteria can continue to multiply during and after the separation process. Under this protocol, the use of pre-packed columns containing $50 \% \mathrm{ZnDPA}$ NPs exhibits a filtering efficiency of $14 \%$. These results highlight that, under the conditions used, flowing cells directly over a pre-packed column result in decreased filtering efficiency compared to batch processes when cells are preincubated with particles for prolonged periods of time and then passed through the magnetic column. The reduction in efficiency is consistent with kinetic and mass transfer limitations that would apply to this single pass filtration process. When NP modification extents are increased to $100 \% \mathrm{ZnDPA}$, the filtering efficiency is increased to $81 \%$. This can be the result of higher NP avidity to cells resulting in more effective cell binding, and highlights how increasing NP modification extents can be used to increase filtering efficacy.

Continuous filtering configurations are advantageous in how scale up can be easily accomplished by increasing the scale of unit operations. To investigate how increasing the number of passes through ZnDPA NP pre-loaded columns affects bacterial filtering efficiency, cells and cell washes were sequentially passed through three columns, while unbound cell count was determined between each step (Fig. 8). After three sequential passes through columns loaded with unmodified FeOA NPs, $25.2 \pm 10$ million CFU was collected, which highlights minimal cell retention when no cell targeting moiety is used. After three sequential passes through 50\% ZnDPA NP columns, the filtering efficiency increased from $13 \%$ on the first pass to $42 \%$ on the third pass, while that of $100 \%$ ZnDPA NP columns increased from $81 \%$ to $93 \%$. This highlights that the efficacy of bacterial removal can be increased by utilizing more columns for separation processes.

\section{Conclusion}

Antimicrobial resistance is among the most pressing problems in healthcare, and the development of new approaches to treat bacterial infections is vital to preserve 


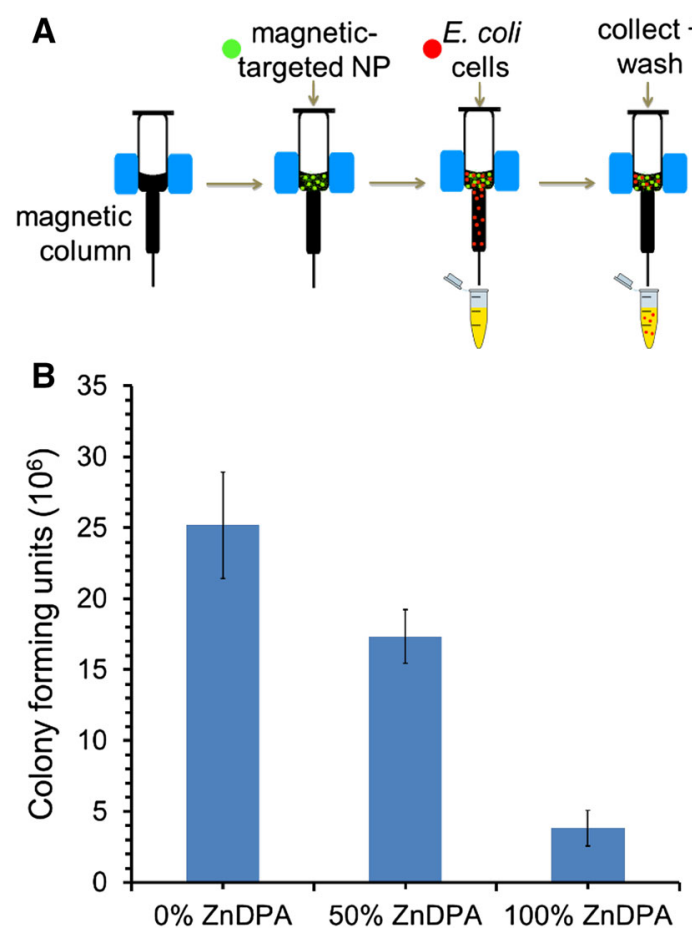

Fig. 7 Batch magnetic separation of cells from media. a Setup of magnetic columns and magnetic ZnDPA NPs to filter $E$. coli from media. Columns are pre-loaded with particles, whereupon cells are loaded and washed off. Effluent cells are collected and counted. b Number of colony forming units collected after $\sim 2 \times 10^{7}$ cells are passed through a column containing ZnDPA iron oxide NPs with varying $\mathrm{ZnDPA}$ modification extents

our capability to treat resistant pathogens. The development of bacterial targeted nanoparticles may be especially useful in improving drug efficacy, drug compliance, drug safety, medical imaging, or even in the bioseparations of bacteria. These constructs must be made in a scalable and cost-effective manner for translation, however, due to the relatively restrictive and low prices of antibiotic treatments. Flash NanoPrecipitation is a scalable and continuous technique of forming nanoparticles, and we here utilize this process to form particles with highly tunable surface properties that target both Gram-positive and Gram-negative pathogens. Importantly, this technique is a platform technology that can be used to encapsulate a variety of core components, such as therapeutics, diagnostic agents, or even magnetically active materials. As a proof-of-principle, we encapsulate fluorescent tracers and utilize iron oxide core loaded particles to bind and magnetically separate bacteria from liquid media. The bacterial separation process is scalable and based on commercially available setups. This work is an example of how nanoparticle-based
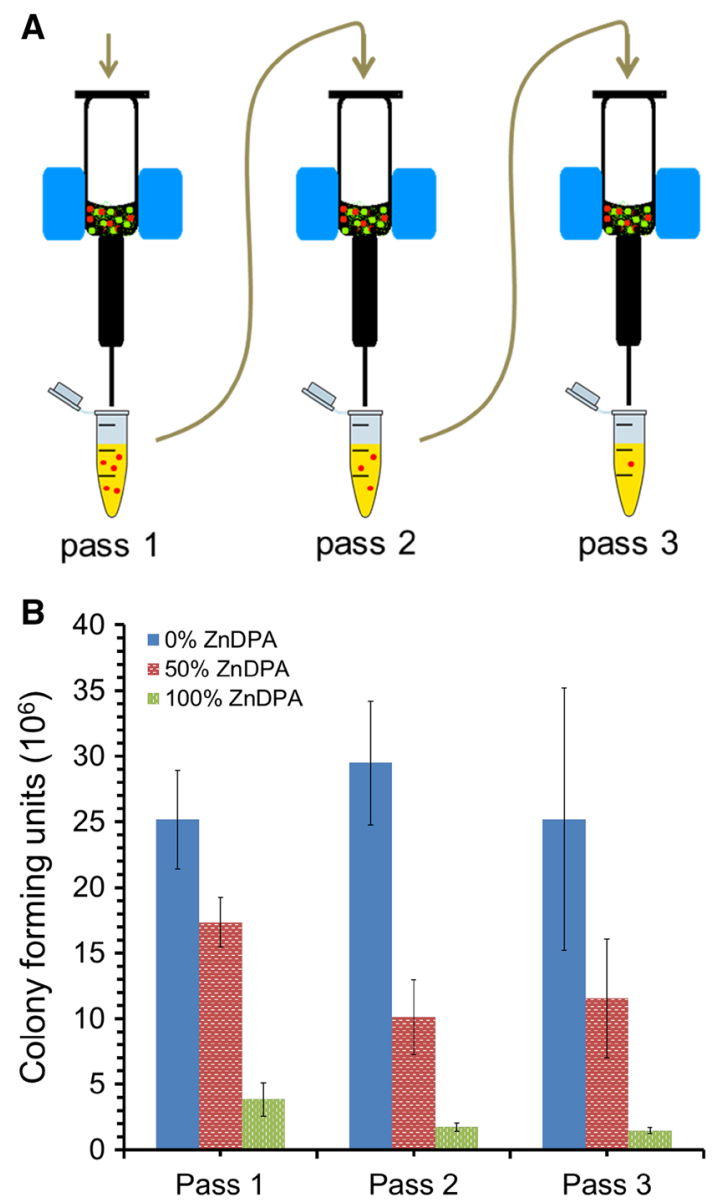

Fig. 8 Serial magnetic separation of cells from media. a Setup of serial magnetic column separation of bacteria. Columns are loaded with particles, whereupon the first column is loaded with cells. Effluent cells are collected and passed on through the subsequent columns. The first column is washed and effluent cells are collected and passed on through subsequent columns. Small volumes of cells between each step is collected, and cells passing through effluent and wash streams throughout each step is the number of colony forming units that pass through the column. b Number of colony forming units collected after $\sim 2 \times 10^{7}$ cells are passed through a column containing ZnDPA iron oxide NPs with varying ZnDPA modification extents

technologies can enable new approaches to combat antibiotic resistant bacterial infections.

Acknowledgements We are grateful for support from the Princeton University Center for Health and Wellbeing (HDL), Woodrow Wilson School of Public and International Affairs Program in Science, Technology, and Environmental Policy (HDL). This work was supported by the Princeton University SEAS grant from the Old School Fund (RKP). We thank Prof. Mark Brynildsen for assistance in the bacterial growth experiments. We thank Christina Decoste and John Grady for assistance on flow cytometry experiments. 
Open Access This article is distributed under the terms of the Creative Commons Attribution 4.0 International License (http:// creativecommons.org/licenses/by/4.0/), which permits unrestricted use, distribution, and reproduction in any medium, provided you give appropriate credit to the original author(s) and the source, provide a link to the Creative Commons license, and indicate if changes were made.

\section{References}

Allen TM (2002) Ligand-targeted therapeutics in anticancer therapy. Nat Rev Cancer 2:750-763. doi:10.1038/nrc903

Bartlett JG, Gilbert DN, Spellberg B (2013) Seven ways to preserve the miracle of antibiotics. Clin Infect Dis 56:1445-1450. doi:10. 1093/cid/cit070

Brown L, Wolf JM, Prados-Rosales R, Casadevall A (2015) Through the wall: extracellular vesicles in Gram-positive bacteria, mycobacteria and fungi. Nat Rev Microbiol 13:620-630. doi:10.1038/nrmicro3480

Cabral $\mathrm{H}$ et al (2011) Accumulation of sub-100 $\mathrm{nm}$ polymeric micelles in poorly permeable tumours depends on size. Nat Nanotechnol 6:815-823. doi:10.1038/nnano.2011.166

Centers for Disease Control and Prevention (2013) Antibiotic Resistance Threats in the United States

Cheng Z, Al Zaki A, Hui JZ, Muzykantov VR, Tsourkas A (2012) Multifunctional nanoparticles: cost versus benefit of adding targeting and imaging capabilities. Science 338:903-910. doi:10. $1126 /$ science. 1226338

Choi SK et al (2013) Dendrimer-based multivalent vancomycin nanoplatform for targeting the drug-resistant bacterial surface. ACS Nano 7:214-228. doi:10.1021/nn3038995

D'Addio SM et al (2012) Effects of block copolymer properties on nanocarrier protection from in vivo clearance. J Control Release 162:208-217. doi:10.1016/j.jconrel.2012.06.020

D'Addio SM et al (2013) Optimization of cell receptor-specific targeting through multivalent surface decoration of polymeric nanocarriers. J Control Release 168:41-49. doi:10.1016/j. jconrel.2013.02.004

Elias DR, Poloukhtine A, Popik V, Tsourkas A (2013) Effect of ligand density, receptor density, and nanoparticle size on cell targeting. Nanomed Nanotechnol Biol Med 9:194-201. doi:10. 1016/j.nano.2012.05.015

Falagas ME, Grammatikos AP, Michalopoulos A (2008) Potential of old-generation antibiotics to address current need for new antibiotics. Expert Rev Anti Infect Ther 6:593-600. doi:10. $1586 / 14787210.6 .5 .593$

Hanshaw RG, Smith BD (2005) New reagents for phosphatidylserine recognition and detection of apoptosis. Bioorg Med Chem 13:5035-5042. doi:10.1016/j.bmc.2005.04.071

Holmes AH, Moore LSP, Steinbakk M, Regmi S, Karkey A, Guerin PJ, Piddock LJV (2016) Understanding the mechanisms and drivers of antimicrobial resistance. Lancet 387:176-187

Johnson BK, Prud'homme RK (2003a) Flash nanoprecipitation of organic actives and block copolymers using a confined impinging jets mixer. Aust J Chem 56:1021-1024

Johnson BK, Prud'homme RK (2003b) Mechanism for rapid selfassembly of block copolymer nanoparticles. Phys Rev Lett 91:118302

Kathleen MM, Ananth A, Ravi VB (2007) Decreased circulation time offsets increased efficacy of PEGylated nanocarriers targeting folate receptors of glioma. Nanotechnology 18:385101
Kell AJ et al (2008) Vancomycin-modified nanoparticles for efficient targeting and preconcentration of Gram-positive and Gramnegative bacteria. ACS Nano 2:1777-1788. doi:10.1021/ nn700183g

Lee H, Fonge H, Hoang B, Reilly RM, Allen C (2010) The effects of particle size and molecular targeting on the intratumoral and subcellular distribution of polymeric nanoparticles. Mol Pharm 7:1195-1208. doi:10.1021/mp100038h

Lee JJ et al (2014) Synthetic ligand-coated magnetic nanoparticles for microfluidic bacterial separation from blood. Nano Lett 14:1-5. doi: $10.1021 / \mathrm{nl} 3047305$

Lu HD et al (2015) Modulating Vibrio cholerae quorum-sensingcontrolled communication using autoinducer-loaded nanoparticles. Nano Lett 15:2235-2241. doi:10.1021/acs.nanolett. 5 b00151

Lu HD, Wilson BK, Heinmiller A, Faenza B, Hejazi S, Prud'homme RK (2016) Narrow absorption NIR wavelength organic nanoparticles enable multiplexed photoacoustic imaging. ACS Appl Mater Interfaces 8:14379-14388. doi:10.1021/acsami.6b03059

Lu HD, Wilson BK, Lim TL, Heinmiller A, Prud'homme RK (2017) Real-time and multiplexed photoacoustic imaging of internally normalized mixed-targeted nanoparticles. ACS Biomater Sci Eng. doi:10.1021/acsbiomaterials.6b00645

Osterberg L, Blaschke T (2005) Adherence to medication. N Engl J Med 353:487-497. doi:10.1056/NEJMra050100

Pansare VJ, Hejazi S, Faenza WJ, Prud'homme RK (2012) Review of long-wavelength optical and NIR imaging materials: contrast agents, fluorophores, and multifunctional nano carriers. Chem Mater 24:812-827. doi:10.1021/cm2028367

Pansare VJ, Bruzek MJ, Adamson DH, Anthony J, Prud'homme RK (2014) Composite fluorescent nanoparticles for biomedical imaging. Mol Imaging Biol 16:180-188. doi:10.1007/s11307013-0689-9

Peer D, Karp JM, Hong S, Farokhzad OC, Margalit R, Langer R (2007) Nanocarriers as an emerging platform for cancer therapy. Nat Nanotechnol 2:751-760. doi:10.1038/nnano.2007.387

Piddock LJV (2012) The crisis of no new antibiotics - what is the way forward? Lancet Infect Dis 12:249-253. doi:10.1016/S14733099(11)70316-4

Plaunt AJ, Harmatys KM, Wolter WR, Suckow MA, Smith BD (2014) Library synthesis, screening, and discovery of modified zinc(II)-Bis(dipicolylamine) probe for enhanced molecular imaging of cell death. Bioconjug Chem 25:724-737. doi:10. $1021 /$ bc500003x

Pustulka KM et al (2013) Flash nanoprecipitation: particle structure and stability. Mol Pharm 10:4367-4377. doi:10.1021/ mp400337f

Rice DR, Gan H, Smith BD (2015) Bacterial imaging and photodynamic inactivation using zinc(II)-dipicolylamine BODIPY conjugates. Photochem Photobiol Sci 14:1271-1281. doi:10.1039/ C5PP00100E

Shen H, Hong S, Prud'homme RK, Liu Y (2011) Self-assembling process of flash nanoprecipitation in a multi-inlet vortex mixer to produce drug-loaded polymeric nanoparticles. J Nanopart Res 13:4109-4120. doi:10.1007/s11051-011-0354-7

Silhavy TJ, Kahne D, Walker S (2010) The bacterial cell envelope. Cold Spring Harb Perspect Biol 2:a000414. doi:10.1101/ cshperspect.a000414

Singh R, Lillard JW (2009) Nanoparticle-based targeted drug delivery. Exp Mol Pathol 86:215-223. doi:10.1016/j.yexmp. 2008.12.004

van Oosten $\mathrm{M}$ et al (2013) Real-time in vivo imaging of invasive- and biomaterial-associated bacterial infections using fluorescently labelled vancomycin. Nat Commun 4:2584. doi:10.1038/ ncomms 3584 
van Oosten M, Hahn M, Crane LMA, Pleijhuis RG, Francis KP, van Dijl JM, van Dam GM (2015) Targeted imaging of bacterial infections: advances, hurdles and hopes. FEMS Microbiol Rev 39:892-916. doi:10.1093/femsre/fuv029

Ventola CL (2015a) The antibiotic resistance crisis. Part 1: Causes and threats. Pharm Ther 40:277-283

Ventola CL (2015b) The antibiotic resistance crisis. Part 2: Management strategies and new agents. Pharm Ther 40:344-352

Viswanathan VK (2014) Off-label abuse of antibiotics by bacteria. Gut Microbes 5:3-4

Wang L, Sun X, Du L, Yuan Q, Li H, Tian H, Li Y (2011) Effects and patient compliance of sustained-release versus immediate-release glipizides in patients with type 2 diabetes mellitus: a systematic review and meta-analysis. J Evid Based Med 4:232-241. doi:10.1111/j.1756-5391.2011.01158.x

Weissmueller NT, Lu HD, Hurley A, Prud'homme RK (2016) Nanocarriers from GRAS zein proteins to encapsulate hydrophobic actives. Biomacromolecules. doi:10.1021/acs.biomac. $6 \mathrm{~b} 01440$
Wisplinghoff H, Bischoff T, Tallent SM, Seifert H, Wenzel RP, Edmond MB (2004) Nosocomial bloodstream infections in US hospitals: analysis of 24,179 cases from a prospective nationwide surveillance study. Clin Infect Dis 39:309-317

Wong PT et al (2015) A lipopolysaccharide binding heteromultivalent dendrimer nanoplatform for Gram negative cell targeting. J Mater Chem B 3:1149-1156. doi:10.1039/C4TB01690D

World Health Organization (2015) Antimicrobial resistance

Zhang L, Gu FX, Chan JM, Wang AZ, Langer RS, Farokhzad OC (2008) Nanoparticles in medicine: therapeutic applications and developments. Clin Pharmacol Ther 83:761-769

Zhang L, Pornpattananangku D, Hu CMJ, Huang CM (2010) Development of nanoparticles for antimicrobial drug delivery. Curr Med Chem 17:585-594

Zhu Z (2014) Flash nanoprecipitation: prediction and enhancement of particle stability via drug structure. Mol Pharm 11:776-786. doi:10.1021/mp500025e 